www.jusletter.ch

Andreas R. Ziegler

\title{
Sind Schweizer Juristen für die Globalisierung gewappnet?
}

\section{Internationale Aspekte in der obligatorischen Ausbildung von Schweizer Juristen}

Internationale Aspekte spielen heute eine immense Rolle in der Tätigkeit der meisten Juristen. Entsprechend ist die Kenntnis, wie man mit diesen Aspekten umgeht, von fundamentaler Wichtigkeit für die zielführende und qualitativ hochstehende Ausbildung von Juristen. In der Schweiz bestehen immense Unterschiede zwischen den vermittelten Inhalten und Fähigkeiten («Skills») (Teil III) an den Ausbildungsstätten und in den unterschiedlichen Programmen (Teil IV). Gewisse Institutionen sollten dringend ihre Minimalanforderungen überdenken (Teil V).

Beitragsart: Beiträge

Rechtsgebiete: Europarecht und Internationales Recht; Forschungs-, Bildungsund Erziehungsrecht

Zitiervorschlag: Andreas R. Ziegler, Sind Schweizer Juristen für die Globalisierung gewappnet?, in: Jusletter 8. Juni 2020 


\section{Inhaltsübersicht}

I. Einleitung

II. Relevante Institutionen und Programme

1. Institutionen

2. Programme

III. Relevante obligatorische Fächer

1. Völkerrecht

2. Europarecht

3. Internationales Privatrecht (und Zivilverfahrensrecht)

4. Rechtsvergleichung

5. Fremdsprachen

6. Internationale Aspekte des schweizerischen Rechts

IV. Stand der Ausbildung (Studienjahr 2019/2020)

1. Université de Lausanne (UNIL) - Ecole de droit
a. Pflichtkurse
b. Fremdsprachen
c. Optionen

2. Universität Luzern (UNILU) - Rechtswissenschaftliche Fakultät
a. Pflichtkurse
b. Fremdsprachen
c. Optionen

3. Universität Freiburg (UNIFR) - Rechtswissenschaftliche Fakultät
a. Pflichtkurse
b. Fremdsprachen
c. Optionen

4. Universität St. Gallen (UNISG) - Law School (LS-HSG)
a. Pflichtkurse
b. Fremdsprachen
c. Optionen

5. Universität Bern (UNIBE) - Rechtswissenschaftliche Fakultät
a. Grundausbildung
b. Fremdsprachen
c. Optionen

6. Universität Zürich $(\mathrm{UZH})$ - Rechtswissenschaftliche Fakultät
a. Pflichtkurse
b. Fremdsprachen
c. Optionen

7. Université de Genève (UNIGE) - Faculté de droit
a. Pflichtkurse
b. Fremdsprachen
c. Optionen

8. Université de Neuchâtel (UNINE) - Faculté de droit
a. Pflichtkurse
b. Fremdsprachen
c. Optionen

9. Universität Basel (UNIBAS) - Juristische Fakultät
a. Pflichtkurse
b. Fremdsprachen
c. Optionen

10. Fernuniversität Schweiz und UniDistance (Brig)
a. Pflichtkurse
b. Fremdsprachen
c. Optionen

11. Zürcher Fachhochschule - ZHAW (Winterthur) 

a. Pflichtkurse
b. Fremdsprachen
c. Optionen

12. HES-SO - HEG Arc (Neuenburg)
a. Pflichtkurse
b. Fremdsprachen
c. Optionen

13. Kalaidos Fachhochschule Schweiz (Zürich)
a. Pflichtkurse
b. Fremdsprachen
c. Optionen

V. Analyse und Postulate

\section{Einleitung}

[1] Internationale Aspekte spielen heute eine immense Rolle in der Tätigkeit der meisten Juristen. Entsprechend ist die Kenntnis, wie man mit diesen Aspekten umgeht, von fundamentaler Wichtigkeit für die zielführende und qualitativ hochstehende Ausbildung von Juristen. In der Schweiz wurde (und wird teilweise) zwar traditionell mehr Gewicht auf diesen Teil der Ausbildung gelegt (Teil II $)^{1}$, doch es bestehen immense Unterschiede zwischen den vermittelten Inhalten und Fähigkeiten («Skills») (Teil III) an den Ausbildungsstätten und in den unterschiedlichen Programmen (Teil IV). Es besteht selten ein klar ausgewiesenes Konzept und sicherlich kein gemeinsames schweizerisches Konzept. Gewisse Institutionen sollten dringend ihre Minimalanforderungen überdenken (Teil V).

[2] Diese Entwicklung wird durch neue Ausbildungsformen (neben dem System BLaw/MLaw, wie es ursprünglich in der Schweiz aus der Bolognareform hervorgegangen ist $)^{2}$ und neue Anbieter (Fernuniversitäten, Fachhochschulen etc.) noch verstärkt. Idealerweise würde die Integration von ausländischem und internationalem (inklusive europäischem) Recht heute in allen Bereichen stattfinden.

[3] Die Fragestellung dieses Beitrags ist natürlich nicht neu. Seit mindestens einhundert Jahren haben sich Juristen mit der Frage schwergetan, wieviel Zeit dem Vermitteln ausländischen und internationalen Rechts zuzugestehen ist. ${ }^{3}$

1 Vgl. Andreas R. Ziegler, Von den Anfängen des Unterrichts des internationalen Rechts in der Schweiz, in: Franco Lorandi / Daniel Staehelin (Hrsg.), Innovative Recht - Festschrift für Ivo Schwander, Zürich 2011, S. 125-140 und Ders., Die Entwicklung der Völkerrechtslehre und -wissenschaft in der Schweiz - eine Übersicht, SZIER 1/2016, S. 21-53.

2 Eine gute Übersicht über die Inhalte der klassischen universitären BLaw und MLaw-Programme findet sich bei Rolf Sethe, Die juristische Ausbildung an den Universitäten der Schweiz: Probleme und Herausforderungen, ZSR 136/2017 II, S. 7-84. Diese geht aufgrund der Konzentration auf den zeitlichen Ablauf des Studiums aber nur am Rande auf die hier behandelte Problematik ein.

3 Vgl. beispielsweise für den deutschen Sprachraum Rudolf Bernhardt (Hrsg.), Das internationale Recht in der Juristenausbildung - Materialien einer Kommission der Deutschen Gesellschaft für Völkerrecht, Heidelberg 1981 oder länderübergreifend; Ryan Scoville / MARK S. BerLin, Who Studies International Law? Explaining Cross-National Variation in Compulsory International Legal Education, EJIL 30/2019, S. 481-508. Aus neuester Zeit auch: Stephan Hobe / Thilo Marauhn (Hrsg.), Lehre des internationalen Rechts im deutschsprachigen Raum - Herausforderungen und Entwicklungspotentiale, in: Hobe / Marauhn (Hrsg.), Lehre des internationalen Rechts - zeitgemäss? Heidelberg 2017, S. 11-34; und darin die Beiträge von DiEs., Lehre des internationalen Rechts im deutschsprachigen Raum - Herausforderungen und Entwicklungspotentiale, S. 11-34. Andrea HamanN, Die Lehre des Völkerrechts in Frankreich - Überblick und Beobachtungen, S. 123-138; LaURI MäLKsoo, Die Lehre des Völkerrechts: ein Blick aus Estland, S. 139-142; Volker RöBen, Die Lehre des Internationalen Rechts in Grossbritannien, S. 143-150. 
Auch in der Schweiz gibt es wenige Beiträge dazu. ${ }^{4}$ Natürlich ist es seit jeher auch ein höchst politisches (und emotionales) Thema, bei dem Nationalisten und Kosmopoliten gegeneinander ausgespielt werden können. ${ }^{5}$ Tatsache ist aber, dass die allgemeinen Folgen der Globalisierung und Integration sich auch in der Rechtswirklichkeit widerspiegeln und entsprechende Kenntnisse gerade heute notwendig sind.

[4] Das sagt für sich allein nichts darüber aus, ob eine rechtliche Lösung besser auf kommunaler, kantonaler, nationaler, europäischer oder globaler Ebene zu finden ist. Aber, das Mehrebenensystem der Rechtsetzung und -anwendung ist eine unbestreitbare Realität ${ }^{6}$, der auch in der Ausbildung Rechnung getragen werden muss. ${ }^{7}$ Selbst wenn man den Staat weiterhin als grundlegenden Baustein der Rechtsordnung akzeptiert, können Fragen des ausländischen und überstaatlichen Rechts nicht ignoriert werden.

[5] Dieses Phänomen mag in grossen Staaten schwächer sein als in einem Kleinstaat mit traditionell intensiven Beziehungen zum Ausland wie der Schweiz. ${ }^{8}$ Hinzu kommen aufgrund der Funktionsweise der internationalen Gesellschaft noch spezifische Fragen des Soft Law bzw. formal nicht verbindlicher Regeln, die zwar auch im innerstaatlichen Recht wichtig sind, auf überstaatlicher Ebene aber noch öfter zu finden sind.

[6] Für die Schweiz von besonderer Bedeutung ist auch eine Resolution der Deutschen Gesellschaft für internationales Recht (in der zahlreiche Schweizer Professorinnen Mitglied sind ${ }^{9}$ ) aus dem Jahre 2016:

«Juristinnen und Juristen müssen sich heute in allen Bereichen nicht mehr nur nationalen und europäischen, sondern auch globalen und transnationalen Herausforderungen stellen. Damit haben sich die Anforderungen an junge Juristinnen und Juristen geändert, von denen fast überall erwartet wird, dass sie sich in einem vom nationalen über das europäische bis hin zum internationalen und transnationalen Recht erstreckenden Ordnungssystem bewegen können. Die Juristenausbildung hat mit dieser Entwicklung nicht im vollen Umfang Schritt gehalten.

Die Deutsche Gesellschaft für Internationales Recht appelliert deshalb an alle Verantwortlichen im deutschen Sprachraum, sich dafür einzusetzen, dass die Grundelemen-

$4 \quad$ Vgl. insbesondere Luzius Wildhaber, Die Bedeutung des Völkerrechts, des Internationalen Privatrechts und der Rechtsvergleichung in der Universitätsausbildung der Juristen in der Schweiz, in: Bernhardt (Fn. 3), S. 99-112 und speziell für die Rechtsvergleichung: Peter V. Kunz, Einführung zur Rechtsvergleichung in der Schweiz - Ein bedeutsames juristisches Fachgebiet für Studenten sowie für Praktiker zwischen «notwendigem Übel» sowie «Königsdisziplin», recht 2/2006, S. 37-54.

5 Vgl. beispielsweise den Einsatz des IKRK, um den Unterricht im humanitären Völkerrecht zu fördern: ETIEnNe Kuster, Promoting the Teaching of IHL in Universities: Overview, Successes, and Challenges of the ICRC's Approach, Journal of International Humanitarian Legal Studies 9/2018, S. 61-97.

6 Vgl. anstelle vieler Beispiele Stephan Breitenmoser / Michel Jutzeler, Schengen und Dublin im Mehrebenensystem, in: Stephan Breitenmoser et al. (Hrsg.), Schengen und Dublin in der Praxis, Zürich 2018, S. 3-32 Nicolas F. Diebold, Freizügigkeit im Mehrebenensystem eine Rechtsvergleichung der Liberalisierungsprinzipien im Binnenmarkt-, Aussenwirtschafts- und Europarecht, Zürich 2016 oder Friederike EnGLER, Steuerverfassungsrecht im Mehrebenensystem - ein Vergleich des Schutzes vor Besteuerung durch EMRK, Grundrechtecharta und die nationale Grundrechtsordnung, Baden-Baden 2014.

7 Auf die innerstaatlichen Aspekte dieser Thematik (Gemeinden, Kantone, Bund) bzw. allgemeinen Fragen des Föderalismus geht der vorliegende Beitrag bewusst nicht ein, obwohl es natürlich systematisch und theoretisch um analoge Fragen geht.

8 Anstelle vieler: Hans Vogel, Der Kleinstaat in der Weltpolitik, Frauenfeld 1979 oder JoËLle Kuntz, La Suisse ou le génie de la dépendance, Carouge-Genève 2013.

9 Der Autor ist im Vorstand dieser Vereinigung. 
te des Völkerrechts, des Internationalen Privatrechts und der Rechtsvergleichung zu einem Bestandteil der juristischen Grundausbildung werden. Im Vertiefungsstudium sollte den Studierenden auch das Angebot gemacht werden, das internationale Recht in fremdsprachlicher und interaktiver Form zu erlernen.

Soweit die Universitäten eine entsprechende Stärkung eigenverantwortlich auf den Weg bringen können, richtet die Gesellschaft diesen Appell an deren Gremien. Darüber hinaus ermuntert die Gesellschaft die juristischen Fakultäten im deutschen Sprachraum, Spezialisierungen im internationalen Recht, einschliesslich qualifizierter Angebote in englischer und ggf. anderen Sprachen zu entwickeln, sowie die Dekanate ggf. um eine Dekanin/einen Dekan für internationale Angelegenheiten zu erweitern.» ${ }^{10}$

\section{Relevante Institutionen und Programme}

\section{Institutionen}

[7] Es wird auf die gemäss Hochschulförderungs- und Koordinationsgesetz (HFKG) eidgenössisch akkreditierten universitären Hochschulen (Basel, Bern, Freiburg, Genf, Lausanne, Luzern, Neuenburg, St. Gallen, Zürich), die Stiftung Universitäre Fernstudien Schweiz, Brig (Fernstudien Schweiz / UniDistance) und die eidgenössisch akkreditierten Fachhochschulen (Zürcher Fachhochschule - ZHAW, HES SO-HEG Arc, Private FH Kalaidos) eingegangen, welche einschlägige Lehrgänge anbieten, die auf eine juristische Tätigkeit vorbereiten sollen. Nicht eingegangen wird auf Lehrangebote, die (völker)rechtliche Komponenten enthalten, aber nicht als juristisch klassifiziert werden können (insbesondere im Bereich Wirtschaft, Staats- oder Politikwissenschaften oder technisch ausgerichteter Studiengänge). Auch auf das (sehr umfangreiche) völkerrechtliche Angebot des Institut de hautes études internationales et du développement (IHEID, Genf), einer Hochschulinstitution gemäss Hochschulförderungs- und Koordinationsgesetz (HFKG), wird daher nicht eingegangen, denn hier wird insbesondere kein Bachelorstudium angeboten. ${ }^{11}$

\section{Programme}

[8] Im Wesentlichen wird auf die klassischen Hauptprogramme Bachelor of Law (180 ECTS ${ }^{12}$, BLaw) und Master of Law (90 ECTS, MLaw) hingewiesen, die nach wie vor von den meisten Stu-

10 Am 16. März 2016 veranstaltete die DGIR an der Universität zu Köln eine Tagung unter dem Titel: «Lehre des internationalen Rechts - zeitgemäss? zum Thema Ausbildung und Lehre im internationalen Recht. Die Teilnehmerinnen und Teilnehmer verabschiedeten dabei die hier wiedergegebene Resolution» (online abrufbar unter: http://www.dgfir.de/veranstaltungen/archiv/lehre-des-internationalen-rechts-zeitgemaess/). (abgerufen: 29. April 2020).

11 Vgl. Graduate Institute, Master \& PhD Programmes, https://graduateinstitute.ch/Master-PhD. Allerdings kann es dazu kommen, dass z.B. ein Bewerber mit einem BLaw einer Schweizer Universität und einem MIL IHEID zur Anwaltsprüfung zugelassen wird, da auch andere Universitäten für ihren MLaw oft volle Wahlfreiheit einräumen. Dies soll hier aber nicht weiter thematisiert werden, da in diesem Fall zumindest eine breite Ausbildung im Völkerrecht sichergestellt wäre (ev. nicht in der vollen Breite, was etwa privat- und europarechtliche Aspekte betrifft).

12 Vgl. Art. 3 der Verordnung des Hochschulrates über die Koordination der Lehre an den Schweizer Hochschulen vom 29. November 2019 (SR 414.205.1): Die Hochschulen und die anderen Institutionen des Hochschulbereichs in der Schweiz wenden das europäische System zur Übertragung und Akkumulierung von Kreditpunkten (Credits; 
dierenden gewählt werden. Dabei wird wo möglich auch angegeben, in welchem Studienabschnitt die Angebote wie angeboten werden. Insbesondere die Angaben zur Aufteilung zwischen Übungen und Vorlesungen oder zur genauen Anzahl Präsenzstunden oder Semesterwochenstunden haben dabei aber nur indikativen Charakter, da hier in der Praxis doch oft relativ viel Flexibilität für die Unterrichtenden besteht. ${ }^{13}$

[9] Wo relevant, wird allerdings auch auf alternative Programme, insbesondere Bachelor of Arts (180 ECTS, BA), Master of Arts (180 ECTS, MA) und Bachelor of Science (180 ECTS, BSc), Master of Science (180 ECTS; MSc), Programme in Recht oder Recht und Wirtschaftswissenschaften (Law and Economics) der Universitäten und Fachhochschulen ${ }^{14}$ eingegangen, insbesondere wenn diese Grundausbildung (Bachelor) den Zugang zu weiterführenden klassischen juristischen Ausbildungen (MLaw) und regulierten Tätigkeiten (Anwalt, Richter, Notar etc.) garantieren soll.

[10] Auf die konsekutiven Masterstudiengänge wird nur insoweit eingegangen, als sie versuchen, in der dazugehörigen Grundstufe Verpasstes nachzuholen. Der «Master of Arts in Legal Studies (MALS)» der Universität Freiburg, der versucht, Nicht-Juristen und Juristen mit einem ausländischen Abschluss fundierte Kenntnisse des Schweizer Rechts zu vermitteln, wird hier nicht näher behandelt. ${ }^{15}$ Genauso wenig werden Doppel- und gemeinsame Abschlüsse auf Masterstufe berücksichtigt, obwohl sie natürlich für die Förderung des Verständnisses der internationalen Aspekte dienlich sind.

[11] Insbesondere wird auch nicht auf die zahlreichen Angebote im Nachdiplombereich (insbesondere CAS, DAS, MAS) eingegangen.

\section{Relevante obligatorische Fächer}

[12] Dabei werden nur die effektiv obligatorischen Fächer angerechnet (Pflichtkurse). Tatsächlich haben gerade die grösseren Fakultäten der Schweiz zumeist ein beeindruckendes fakultatives Angebot in diesem Bereich. Man stellt aber fest, dass es eben grösstenteils nur als Option im ohnehin zumeist sehr frei gestalteten Vertiefungsbereich (v.a. auch auf Masterstufe) angeboten wird. Damit kann nicht garantiert werden, dass der Absolvent effektiv in den betreffenden Gebieten geschult wurde. Häufig sind es ganz wenige besonders interessierte Studierende, die eine Tätigkeit im internationalen Bereich anstreben oder solche aus dem Ausland (im Austausch oder hier für ein Masterstudium), die diese Kurse in grosser Anzahl belegen. Der Vollständigkeit halber wird aber dennoch kursorisch auf diese Angebote hingewiesen (Optionen, Wahlfächer, Wahlpflichtfächer, die ganz vermieden werden können). Dasselbe gilt für Fremdsprachenange-

ECTS) an. Sie vergeben für überprüfte Studienleistungen Credits. Ein Credit entspricht einem Arbeitsaufwand von 25-30 Stunden.

13 Vgl. den Versuch einer umfassenden (aber nicht ganz korrekten und bereits nicht mehr ganz aktuellen) Darstellung bei Sethe (Fn. 2), S. 37-38.

14 Vgl. dazu Art. 11 der Verordnung des Hochschulrates über die Koordination der Lehre an den Schweizer Hochschulen vom 29. November 2019 (SR 414.205.1). An dieser Stelle soll nicht näher auf die Anerkennung bzw. den Übertritt in ein universitäres Masterstudium (MLaw) mit einem Bachelor einer Fachhochschule eingegangen werden, es sei denn, dabei spielten Fragen des obligatorischen Unterrichts in internationalen Aspekten des Rechts eine tragende Rolle.

15 ER scheint aber keine obligatorischen Vorlesungen zu internationalen Aspekten zu enthalten: Universität Freiburg i. Ü., Ius, Programme des cours https://www3.unifr.ch/ius/fr/etudes/coursexamens/cours/ (abgerufen: 29. April 2020). 
bote, die ebenfalls recht umfassend angeboten werden, aber noch eher selten Pflicht sind, womit sie ebenfalls praktisch ganz einfach vermieden werden können (und in der Realität auch werden).

[13] Für den folgenden Vergleich wurden einige Disziplinen als besonders relevant zu Grunde gelegt: Völkerrecht, Europarecht, Rechtsvergleichung (und spezielle rechtsvergleichende Fächer wie Privatrechtsvergleichung, vergleichendes Verfassungsrecht etc.) sowie Fremdsprachen. ${ }^{16}$

\section{Völkerrecht}

[14] Das Völkerrecht bzw. das internationale (öffentliche) Recht wird in der Schweiz in der Juristenausbildung zumeist noch in einem gesonderten Kurs unterrichtet. ${ }^{17}$ Dabei gilt es aber zu beachten, dass Teile davon schon heute in der Grundausbildung auch in anderen Kursen behandelt werden können. Ein besonders gutes Beispiel dafür ist die Tatsache, dass der Grundrechtsschutz (normalerweise in obligatorischen Vorlesungen zum Verfassungsrecht) kaum sinnvoll ohne Bezüge zum internationalen und regionalen (europäischen) Recht unterrichtet werden kann (insbesondere EMRK). Dennoch wird in der folgenden Darstellung darauf verzichtet, die obligatorischen Vorlesungen im öffentlichen Recht darauf zu untersuchen, wieweit sie internationale Quellen (insbesondere im Grundrechtsschutz) behandeln.

\section{Europarecht}

[15] Daneben umfasst die vorliegende Untersuchung das heute für die Schweiz eng dazu gehörende Europarecht ${ }^{18}$, welches zumeist auf das Recht der Europäischen Union (EU) ausgerichtet ist und teilweise auch mit den bilateralen Rechtsbeziehungen kombiniert wird. Dabei stellt die Schweiz einen Drittstaat dar. Nur selten werden hierbei auch Quellen anderer europäischer Organisationen (insbesondere Europarat oder EFTA) behandelt. ${ }^{19}$

\section{Internationales Privatrecht (und Zivilverfahrensrecht)}

[16] Ausserdem besteht hierzulande eine lange Tradition, gewisse internationale Aspekte des Privatrechts (insbesondere das Kollisionsrecht) in eigene Vorlesungen unter dem Titel Internationales Privatrecht (IPR), allenfalls mit besonderer Erwähnung von verfahrensrechtlichen Aspekten (Internationales Zivilprozessrecht - IZPR) zu unterrichten. ${ }^{20}$

16 Tatsächlich stellt dies eine Vereinfachung dar, denn natürlich können bereits heute internationale Aspekte in anderen Fächern abgehandelt werde. In der obligatorischen Grundausbildung stellt das aber die Ausnahme dar (v.a. mit Ausnahme des internationalen Menschenrechtsschutzes im Bereich des öffentlichen Rechts oder gewisser Aspekte der Einführung in die Rechtwissenschaft).

17 Vgl. zur Entwicklung des Unterrichts im Völkerrecht in der Schweiz: vgl. ZIEGLeR (Fn.1). Für andere Länder HoBe / Marauhn (Fn 2).

18 Vgl. zur Entwicklung des Unterrichts im Europarecht in der Schweiz ZIEGLER (Fn 1).

19 Vgl. zur Charakterisierung und Abgrenzung die gängigen Lehrbücher zum Europarecht für die Schweiz, z.B. Matthias Oesch, Europarecht. Band I: Grundlagen, Institutionen, Verhältnis Schweiz-EU, 2. Aufl., Bern 2019.

20 Vgl. Marc-Philippe Weller, Die Lehre des Internationalen Privatrechts im deutschsprachigen Raum - Herausforderungen und Entwicklungspotentiale, in: Hobe / Marauhn (Fn. 2), S. 35-48. 


\section{Rechtsvergleichung}

[17] Auch die Rechtsvergleichung spielt in der Schweiz traditionell eine wichtige Rolle, um den überstaatlichen Kontext und mögliche Konflikte zwischen staatlichen Rechtsordnungen besser zu erkennen. ${ }^{21}$ Dabei trifft man zumeist den Unterricht entweder auf der Grundlage einer eher theoretischen Ausrichtung (Rechtsvergleichung, insbesondere mit Bezügen zum Privatrecht) oder aber direkt auf ein Rechtsgebiet ausgerichtet an (v.a. vergleichendes Verfassungsrecht neben der Privatrechtsvergleichung). Zunehmend ist auch wieder der thematisch ausgerichtete Unterricht, bei dem internationale, europäische und vergleichende Aspekte kombiniert werden, anzutreffen, so wie er in den Anfängen der diesbezüglichen institutionalisierten Forschung häufig war. ${ }^{22}$ Dies ist allerdings in der Schweiz zumindest in der Grundausbildung bzw. im obligatorischen Unterricht selten bis nie der Fall. Nur selten sind auf dieser Stufe bereits obligatorische Vorlesungen zu wichtigen Spezialgebieten wie dem Internationalen Strafrecht, dem Internationalen Steuerrecht etc. zu finden. ${ }^{23}$

[18] Wenn wir die moderne universitäre Juristenausbildung in der Schweiz seit dem 19. Jahrhundert zugrunde legen, wurden diese Aspekte im Kleinstaat Schweiz von Anfang an grundsätzlich anerkannt. Bei der Entwicklung des innerstaatlichen Rechts wurde dabei ein ansehnlicher Stand in der Rechtsvergleichung erreicht, der zumindest teilweise auch in die Juristenausbildung einfloss, anfänglich insbesondere im Privatrecht, später auch im öffentlichen Recht (inklusive Strafrecht). Mit dem Abschluss der grundlegenden Kodifizierungen konnte man aber vielerorts eine Reduktion, ja geradezu Verarmung auf diesem Gebiet feststellen, was insbesondere von den Vertretern einer praxisnahen Ausbildung befürwortet wurde. Die Möglichkeit der zeitweisen Ausbildung im Ausland (Austauschprogramme, Postgraduiertenprogramme, gemeinsame Ausbildungsgänge) konnte den Folgen dieser Tendenz nur beschränkt etwas entgegensetzen. Im Privatrecht spielte lange Zeit das römische Recht (und die Rechtsgeschichte) eine wichtige Funktion, um aktuelle Institutionen und Normen auf gemeinsame klassische Vorläufer zurückzuführen. Im vorliegenden Beitrag soll darauf aber nicht weiter eingegangen werden. ${ }^{24}$

\section{Fremdsprachen}

[19] Eine spezielle Thematik stellt in vielen Ausbildungslehrgängen die Frage dar, inwieweit Fremdsprachenkenntnisse gefördert werden müssen. Oft wird dies zwar grundsätzlich begrüsst, aber die Frage, ob spezifische Kurse (Fachterminologie) angeboten werden müssen und ob diese als Studienleistungen anerkannt werden, ist zumeist sehr kontrovers. Im Folgenden soll der Be-

21 Vgl. zu diesem Aspekt v.a. Kunz (FN. 3).

22 Vgl. etwa das Kaiser-Wilhelm-Institut für ausländisches öffentliches Recht und Völkerrecht (gegründet 1924), das Kaiser-Wilhelm-Institut für ausländisches und internationales Privatrecht (gegründet 1926) oder die Zeitschrift «International and Comparative Law Quarterly» (gegründet 1952). Das Schweizerische Institut für Rechtsvergleichung (SIR) in Lausanne trägt zwar einen kürzeren Namen, das Institut ist aber eine Dokumentations- und Forschungsstätte für Rechtsvergleichung, ausländisches und internationales Recht (Art. 2 Bundesgesetz über das Schweizerische Institut für Rechtsvergleichung (SIRG) vom 28. September 2018 SR 425.1).

23 Wo dies ausnahmsweise der Fall ist, wird im Folgenden aber darauf hingewiesen.

24 Neben der allgemeinen Diskussion zur Rolle des römischen Rechts (und zu einem Obligatorium ev. sogar mit Kenntnissen in Latein) sei hier aber auf ein fakultatives Angebot etwa an der Universität Lausanne hingewiesen. Dort unterrichtet Professor Hansjörg Peter einen Kurs Fondements communs du droit européen (4 ECTS, 56 Stunden Präsenzunterricht) auf Bachelorstufe, der neben dem obligatorischen Unterricht im römischen Recht und der Rechtsgeschichte angeboten wird. 
stand an solchen Unterrichtsformen aufgeführt werden, da die Frage für die Schweiz einerseits wegen ihrer Vielsprachigkeit eine besondere Relevanz hat und zudem der Zugang zum ausländischen und überstaatlichen Recht ohne gewisse Fremdsprachen (insbesondere Englisch) heute nicht mehr ohne Weiteres möglich ist. Die Tatsache, dass technologische Hilfsmittel hierbei die Situation in Zukunft noch stärker verändern könnten (Legal Tech), führt allein noch nicht dazu, dass zum Verständnis und zur aktiven Teilnahme an der Rechtsentwicklung die entsprechenden Sprachkenntnisse nicht essenziell bleiben.

\section{Internationale Aspekte des schweizerischen Rechts}

[20] Eine besondere Problematik besteht darin, dass der Erwerb internationaler Aspekte des Rechts im Ausland zwar grundsätzlich sehr wünschenswert und positiv zu bewerten ist. Er garantiert aber nicht das Verständnis, wie die schweizerische Rechtsordnung genau mit dem Völkerrecht oder Europarecht umgeht (Rang, unmittelbare Anwendbarkeit, Berücksichtigung der Rechtsprechung des EuGH etc.), oder wie das schweizerische IPR oder IZPR sich darstellt (z.B. ordre public in der Rechtsprechung des Bundesgerichts oder Fragen des genügenden Binnenbezugs bei der Anerkennung von ausländischen Entscheiden oder Schiedsurteilen). Auf diese Problematik soll hier nicht näher eingegangen werden (da sie sich zumeist nicht in den Lehrprogrammen spiegelt, wird aber im Rahmen der Postulate am Ende dieses Beitrages aufgenommen.

\section{Stand der Ausbildung (Studienjahr 2019/2020) \\ 1. Université de Lausanne (UNIL) - Ecole de droit \\ a. Pflichtkurse}

[21] Das umfangreichste Pflichtprogramm in internationalen Fächern (20 ECTS) und Fremdsprachen (3 ECTS) aller hier untersuchten Institutionen hat zurzeit die Ecole de droit der Universität Lausanne. ${ }^{25}$ Hier werden im Bachelorstudium (BLaw) drei Pflichtkurse in den Fächern Völkerrecht (erstes Studienjahr, Droit international public, 6 ECTS mit 56 Stunden Präsenzunterricht 2 SWS während des 1. und 2. Semesters), Europarecht (2. oder 3. Studienjahr, Droit européen, 6 ECTS mit 56 Stunden Präsenzunterricht - 4 SWS während eines Semesters) und Internationales Privatrecht/Rechtsvergleichung (2. oder 3. Studienjahr; Droit comparé et Droit international privé, 8 ECTS mit 84 Stunden Präsenzunterricht - 3 SWS während 2 Semestern) vorgeschrieben. Damit resultiert ein Minimum von 20 ECTS Unterricht in internationalen Fächern mit garantiertem Absolvieren der Fächer Völkerrecht, Europarecht, Rechtsvergleichung und Internationales Privatrecht (IPR). ${ }^{26}$

25 Vgl. Université de Lausanne, Faculté de droit, des sciences criminelles et d'administration publique : Plan d'études (BLaw) 2019, https://www.unil.ch/ecolededroit/fr/home/menuinst/enseignement/bachelor-en-droit/ reglements--plans-detudes.html. (abgerufen: 29. April 2020).

26 Vgl. zur Entwicklung Andreas R Ziegler / Jérome ReYmond, L'enseignement lausannois du droit international public, de Barbeyrac à nos jours : un exemple de diversification, in: Denis Tappy / Bettina Kahil-Wolff / Léonard Breuchez, (Hrsg.), 300 ans d'enseignement de droit à Lausanne ( = Mélanges offertes en honneur du Tricentennaire de la Faculté de droit et des sciences criminelles de l’Université de Lausanne), Zürich 2010, S. 421-441. 


\section{b. Fremdsprachen}

[22] Obligatorisch wird der Erwerb von 3 ECTS (2 SWS während zwei Semestern, 56 Stunden Präsenzunterricht insgesamt) in deutscher Rechtssprache (Langue juridique allemande) verlangt.

c. Optionen

[23] Im Bachelor besteht die Option, den Kurs im Sozialrecht alternativ auf Deutsch zu belegen. Im Masterstudium besteht wie an den meisten juristischen Fakultäten gegenwärtig noch freie Kurswahl. Dabei kann u.a. eine Spezialisierung in Droit international et comparé erworben werden. Sie wird von zahlreichen ausländischen Studenten gewählt, denen der Zugang zu den auf das schweizerische Recht fokussierten Studiengängen verwehrt ist. Hinzuweisen ist zudem auf das immer noch bestehende Spezialprogramm im deutschen Recht, das aber im Wesentlichen von deutschen Studenten im Austausch genutzt wird. Hinzu kommen englischsprachige Weiterbildungprogramme wie etwa ein LLM in International Business Law (MAS).

\section{Universität Luzern (UNILU) - Rechtswissenschaftliche Fakultät a. Pflichtkurse}

[24] An der Universität Luzern ${ }^{27}$ werden zurzeit im Bachelorstudium die internationalen Fächer «Völkerrecht» (4 ECTS, 28 Stunden Präsenzunterricht und 12 Stunden Übungen, 2 SWS im 2. Semester $)^{28}$ und «Europarecht» ${ }^{29}$ (4 ECTS, 28 Stunden Präsenzunterricht, 2 SWS im 3. Semester) vorgeschrieben. Ein eigenes Fach «Rechtsvergleichung» oder eine Vorlesung zur Privatrechtsvergleichung oder zum vergleichenden Verfassungsrecht werden obligatorisch ebenso wenig vorgeschrieben wie das IPR, welches früher noch im Bachelor unterrichtet wurde. ${ }^{30}$ Damit resultieren insgesamt 8 ECTS in internationalen Fächern.

\section{b. $\quad$ Fremdsprachen}

[25] Der obligatorische Erwerb von juristischer Terminologie einer Fremdsprache wird nicht mehr verlangt. In den Anfängen der Fakultät wurden noch Legal English, Introduction to AngloAmerican Legal Thinking, Français Juridique oder «Deutsche Rechtssprache» (für Italienischsprachige) im Grundstudium geprüft.

27 Vgl. Universität Luzern, Fakultät III für Rechtswissenschaft: Studien- und Prüfungsordnung vom 28. September 2016 (https://www.unilu.ch/fileadmin/fakultaeten/rf/0_Dekanat_RF/Dok/reglemente/Studien__und_Pruefungsordnung_2016.pdf) und Musterstudienplan (https://www.unilu.ch/studium/lehrveranstaltungen-pruefungenreglemente/rf/reglemente/\#section=c75058). (abgerufen: 29. April 2020).

28 Vgl. Universität Luzern, Öffentliches Recht (Völkerrecht), https://vv.unilu.ch/details?code=FS201159 (abgerufen: 29. April 2020).

29 Vgl. Universität Luzern, Öffentliches Recht (Europarecht), https://vv.unilu.ch/details?code=HS191039 (abgerufen: 29. April 2020).

30 Vgl. Studien- und Prüfungsordnung (StuPO 2011): https://www.unilu.ch/studium/ lehrveranstaltungen-pruefungen-reglemente/rf/reglemente/\#section=c34921 (abgerufen: 29. April 2020). 
c. Optionen

[26] Auf Masterstufe besteht erneut völlige Wahlfreiheit ${ }^{31}$. Hier wird etwas das IPR (5 ECTS) angeboten, aber auch Internationales Familien- und Erbrecht (5 ECTS), eine Introduction to the Common Law System (5 ECTS) etc. ${ }^{32}$ Ausserdem wird u.a. auch ein Masterprofil «Internationales Recht \& Menschenrechte» angeboten.

\section{Universität Freiburg (UNIFR) - Rechtswissenschaftliche Fakultät a. Pflichtkurse}

[27] An der Universität Fribourg wird von der Rechtswissenschaftlichen Fakultät auf Bachelorstufe (BLaw) $)^{33}$ obligatorisch ein Kurs «Europa- und Völkerrecht» im Umfang von 9 ECTS zur Absolvierung im ersten Studienjahr vorgeschrieben. Die Kurse werden parallel auf Deutsch und Französisch angeboten. Während zweier Semester (1. Studienjahr) werden dabei jeweils 2 SWS Vorlesungen und 4 SWS Übungen absolviert (168 Stunden Präsenzunterricht insgesamt). Internationales Privatrecht und Rechtsvergleichung werden auf dieser Stufe nicht geprüft. ${ }^{34}$ Es resultieren also 9 ECTS obligatorisch in internationalen Aspekten.

\section{b. Fremdsprachen}

[28] Bezüglich des Fremdsprachenunterrichts ist es möglich im Rahmen des BLaw, optional den Zusatz «Zweisprachig» zu erlangen, indem während des Bachelor-Studiums mindestens 72 ECTSKreditpunkte in der Zweitsprache (Deutsch oder Französisch) erworben werden. Dazu gehört auch das Verfassen einer Proseminararbeit in der anderen Unterrichtssprache. Alternativ kann ein ganzer Prüfungsblock und eine Proseminararbeit in der Zweitsprache absolviert werden, um diesen Zusatz zu erlangen. Die Zweisprachigkeit ist aber rein optional. Rechtsenglisch wird auf dieser Stufe weder verlangt noch angeboten.

\section{c. Optionen}

[29] Optional kann auf Bachelorstufe (BLaw) bereits ein Zusatz «Europarecht» erlangt werden, wenn zusätzlich die «Einführung in das europäische Privatrecht» (4 ECTS) und der Kurs «Binnenmarktrecht» (4 ECTS) sowie eine Proseminararbeit im Umfang von 3 ECTS zu einem Thema des Europarechts absolviert wird. Auf der Masterstufe herrscht freie Fächerwahl mit der Mög-

31 Vgl. § 19 der Studien- und Prüfungsordnung der Rechtswissenschaftlichen Fakultät der Universität Luzern vom 28. September 2016, https://www.unilu.ch/studium/lehrveranstaltungen-pruefungen-reglemente/rf/reglemente/ \#section=c34921 (abgerufen: 29. April 2020).

32 Vgl. Universität Luzern Masterprogramm: https://www.unilu.ch/studium/ lehrveranstaltungen-pruefungen-reglemente/rf/lehrveranstaltungen/\#section=c14662 (abgerufen: 29. April 2020).

33 Vgl. Université de Fribourg, Faculté de droit, Règlement du 28 juin 2006 (Etat le 10 décembre 2018) des études de droit, online : https://www3.unifr.ch/apps/legal/fr/document/790199 (abgerufen: 29. April 2020).

34 Vgl. Université de Fribourg: Programme des cours (https://www3.unifr.ch/timetable/fr/) (abgerufen: 29. April 2020). 
lichkeit zur Vertiefung auch im Europarecht. ${ }^{35}$ In Nachdiplombereich werden verschiedene internationale Programme angeboten.

[30] Daneben bietet die Wirtschafts- und Sozialwissenschaftliche Fakultät (in Zusammenarbeit mit der Rechtswissenschaftlichen Fakultät) ein Studienangebot «Bachelor of Arts (BA) in Wirtschafts- und Rechtswissenschaftlichen Studien». Die Studierenden absolvieren zwei Drittel ihres Studiums an der Wirtschafts- und Sozialwissenschaftlichen Fakultät (Hauptfach in «Management und Ökonomie», 120 ECTS-Kreditpunkte) und das restliche Drittel an der Rechtswissenschaftlichen Fakultät (Grosses Nebenfach in «Rechtswissenschaften», 60 ECTS- Kreditpunkte). Gemäss Reglement für Studierende mit Rechtswissenschaften als Nebenfach können sich die Studierenden ihr Programm an der Rechtswissenschaftlichen Fakultät während ihres gesamten Bachelorstudiums selbst zusammenstellen. Es bestehen also auch keine Pflichtleistungen im internationalen Recht. Aufgrund seiner starken wirtschaftswissenschaftlichen Ausrichtung wird er nur ausnahmsweise (mit Auflagen) mit einem BLaw gleichzusetzen sein, wenn ein MLawStudium oder die Zulassung zu einer Tätigkeit, für die normalerweise ein BLaw vorausgesetzt wird, angestrebt werden. ${ }^{36}$

\section{Universität St. Gallen (UNISG) - Law School (LS-HSG)}

\section{a. Pflichtkurse}

[31] An der Universität St. Gallen ${ }^{37}$ werden im Rahmen des klassischen BLaw im 5. Semester 3 ECTS im «Völkerrecht» und 3 ECTS im «Europarecht» obligatorisch erworben, im 6. Semester zusätzlich 3 ECTS «Internationales Privatrecht» (je 21 Stunden Präsenzunterricht), damit resultiert ein Pflichtprogramm von 9 ECTS in internationalen Fächern, was 63 Stunden Präsenzunterricht entspricht. Rechtsvergleichung wird auf dieser Stufe nicht angeboten. ${ }^{38}$

[32] Die Law School der Universität St. Gallen bietet zusätzlich einen Bachelor of Arts in Law and Economics (BA HSG in Rechtswissenschaft mit Wirtschaftswissenschaften), der wie ein BLaw (nach Abschluss des Masterstudiums) den Zugang zum Anwaltspraktikum garantieren soll. In diesem Programm verzichtet man ganz auf Vorlesungen im Völker- und Europarecht. Es wird auf Bachelorstufe lediglich die Vorlesung «Internationales Privatrecht» im 6. Semester (3 ECTS, 21 Stunden Präsenzunterricht) obligatorisch absolviert. ${ }^{39}$

35 Vgl. Université de Fribourg, Faculté de droit, Règlement du 28 juin 2006 (Etat le 10 décembre 2018) des études de droit, online : https://www3.unifr.ch/apps/legal/fr/document/790199 (abgerufen: 29. April 2020). Vgl. hierzu die Bemerkungen zum BLE der Universität St. Gallen (Teil IV.4), bei dem der Zugang zum Anwaltspatent explizit in Aussicht gestellt wird.

37 Vgl. Universität St. Gallen: https://www.unisg.ch/de/studium/bachelor/majorrechtswissenschaft/curriculum (abgerufen: 29. April 2020).

38 In St. Gallen soll anscheinend eine Studienreform auf den Weg gebracht worden sein, die für den BLaw und BLE 2021 in Kraft treten könnte. Danach würden das Völkerrecht und das Europarecht nicht mehr für alle BachelorStudierenden obligatorisch sein, sondern Teil eines «Pflichtwahlbereichs» Internationales Recht werden, wobei beide Vorlesungen auf je 4 ECTS angehoben würden. Die Reform schafft insgesamt sechs «Pflichtwahlkörbe» (Gesellschaftsrecht, Internationales Recht, Verfahrensrecht, Verfassungsrecht, Strafrecht, Wirtschaftsrecht), zwischen denen die Studierenden wählen können (während die sog. «Kernfächer» obligatorisch sind). Innerhalb jedes Pflichtwahlkorbs gibt es wiederum eine begrenzte Wahlfreiheit; so sollen die Studierenden im Pflichtwahlbereich Internationales Recht aus mindestens sechs Kursangeboten mindestens drei wählen. Damit würden 12 ECTS resultieren. Provisorische Auskunft von Prof. Bardo Fassbänder vom 28. April 2020).

39 Entsprechend lässt z.B. die Universität Zürich solche BLE-Absolventen nur mit Auflagen zu ihren MLawProgrammen zu: «Studierende mit einem Bachelor of Law \& Economics der Universität St. Gallen werden zu 
b. Fremdsprachen

[33] Fremdsprachenunterricht ist an der Universität St. Gallen für alle Studenten im Rahmen des Assessmentjahres (1. Jahr) obligatorisch vorgesehen. Da der Rechtswissenschaftliche Lehrgang nur auf Deutsch begonnen werden kann, sind dabei 4 ECTS in einer Fremdsprache zu erwerben. Dabei handelt es sich nicht notwendigerweise um juristische Terminologie. Die Universität empfiehlt angehenden Juristen im Rahmen dieses Obligatoriums die Wahl des Kurses English for Law. ${ }^{40}$ Daneben besteht auch die Möglichkeit, stattdessen den Kurs Le français du droit zu absolvieren. ${ }^{41}$

c.

\section{Optionen}

[34] Auf Masterstufe werden zahlreiche Kurse angeboten, wobei auch Fremdsprachen eingesetzt werden können (v.a. Englisch). ${ }^{42}$ Daneben besteht an der School of Economics and Political Science (SEPS-HSG) ein eigenständiges Master-Programm in International Law (MIL), welches auf Englisch angeboten wird. Auf dieser Stufe holen Master of Law and Economics-Studenten einen Kurs «Völker- und Europarecht für MLE» nach. Da die Absolvierung beider Studienstufen aber nicht garantiert ist, wird sie hier nicht als obligatorisch bewertet.

\section{Universität Bern (UNIBE) - Rechtswissenschaftliche Fakultät \\ a. Grundausbildung}

[35] An der Universität Bern ${ }^{43}$ wird im Rahmen der Vorlesung «Öffentliches Recht I: Einführung in das Völkerrecht und Verwaltungsrecht» im 2. Semester (4 SWS Vorlesung, 2 SWS Übungen, 9 ECTS) auch eine Einführung in das Völkerrecht obligatorisch absolviert (ca. 42 Stunden Präsenzunterricht, 4.5 ECTS). Im dritten Semester wird die Vorlesung «Öffentliches Recht II: Staatsorganisationsrecht und Einführung in das Europarecht» (2 SWS, insgesamt 28 Stunden Präsenzunterricht, 3 ECTS) absolviert, wobei erneut angenommen werden muss, dass nur die Hälfte dem Europarecht zugerechnet werden kann (1 SWS, insgesamt 14 Stunden Präsenzunterricht 1.5 ECTS insgesamt). Es besteht kein obligatorisches Angebot auf dieser Stufe im IPR oder in Rechtsvergleichung. Es kann also von einem Pflichtprogramm von ca. 6 ECTS in internationalen Fächern ausgegangen werden.

den Studiengängen Master of Law UZH, Master of Law UZH mit Schwerpunkt Rechtspraxis sowie Master of Law UZH mit Schwerpunkt Öffentliches Recht mit Auflagen im Umfang von 15 ECTS Credits (Transnationales Recht mit 12 ECTS Credits und Fallbearbeitung der Aufbaustufe im Privatrecht mit 3 ECTS Credits) zugelassen.», vgl. https://www.ius.uzh.ch/de/studies/general/admission.html (abgerufen: 29. April 2020).

40 Vgl. Universität St. Gallen: https://www.unisg.ch/de/studium/bachelor/assessmentjahr/curriculum/ kontextstudium/fremdsprachen/englisch (abgerufen: 29. April 2020).

41 Vgl. Universität St. Gallen: https://www.unisg.ch/de/studium/bachelor/assessmentjahr/curriculum/ kontextstudium/fremdsprachen/franzoesischlacommunicationprofessionnelle (abgerufen: 29. April 2020).

42 Vgl. Universität St. Gallen : http://www.unisg.ch/de/studium/bachelor/majorrechtswissenschaft/curriculum/ pflichtbereich (abgerufen: 29. April 2020).

43 Vgl. Studienplan für das Bachelor-Monoprogramm und das Master-Monoprogramm in Rechtswissenschaft vom 16.10.2014 und Anhänge zum Studienplan für das Bachelor-Monoprogramm und das Master-Monoprogramm in Rechtswissenschaft vom 16. Oktober 2014 (Stand 1. August 2019): https://www.rechtswissenschaft.unibe.ch/ studium/studienprogramme/bachelor_rechtswissenschaft/index_ger.html (abgerufen: 29. April 2020). 


\section{b. Fremdsprachen}

[36] Es bestehen keine Pflichtangebote in Fremdsprachen.

\section{c. Optionen}

[37] Auf Masterstufe besteht weitestgehend Wahlfreiheit, wobei zahlreiche Kurse angeboten werden und auch Fremdsprachen eingesetzt werden können.

\section{Universität Zürich (UZH) - Rechtswissenschaftliche Fakultät a. Pflichtkurse}

[38] An der Universität Zürich ${ }^{44}$ werden die internationalen Fächer im Aufbaustudium angeboten (2. und 3. Jahr des BLaw). Das «Modul Völkerrecht/Europarecht» erstreckt sich über 2 Semester und umfasst die Veranstaltungen «Völkerrecht» (Herbstsemester, 3 ECTS, 2 SWS über ein Semester, 28 Stunden Präsenzunterricht) und «Europarecht/Institutionen» (Frühjahrssemester, 3 ECTS, 2 SWS über ein Semester, 28 Stunden Präsenzunterricht). Im Rahmen der geplanten Studienreform $2021^{45}$ würde dieser Bereich von 6 auf 9 ECTS aufgewertet). Im Modul «Internationales Privat- und Zivilverfahrensrecht» sind die Rechtsgebiete IPR und IZPR zusammengefasst (6 ECTS, 2 SWS über zwei Semester, insgesamt 56 Stunden). Es würde auch nach der Studienreform im gleichen Rahmen angeboten. Damit resultieren zurzeit 12 ECTS in internationalen Fächern, während nach der Studienreform 15 ECTS verlangt würden.

\section{b. Fremdsprachen}

[39] Es besteht keine Pflicht, Fremdsprachen zu lernen oder Kurse in Fremdsprachen zu belegen.

\section{c. Optionen}

[40] Insbesondere auf Masterstufe wird ein grosses Angebot an internationalen Fächern angeboten, auch Doppelabschlüsse und gemeinsame Abschlüsse mit interkulturellem und internationalem Charakter. Zudem bestehen Nachdiplomangebote.

44 Vgl. Universität Zürich: Rechtswissenschaftliche Fakultät, Bachelorstudium: https://www.ius.uzh.ch/de/studies/ bachelor.html (abgerufen: 29. April 2020).

45 Vgl. Universität Zürich: Rechtswissenschaftliche Fakultät, Bachelorstudium (Bologna): https://www.ius.uzh.ch/de/ studies/bologna21/bachelor.html (abgerufen: 29. April 2020). 


\section{Université de Genève (UNIGE) - Faculté de droit a. Pflichtkurse}

[41] An der Universität Genf ${ }^{46}$ werden das Völkerrecht (Droit interntional public, 2 SWS im 2. Jahr, 6 ECTS, 56 Stunden Präsenzunterricht), das Internationale Privatrecht (Droit international privé, 5 ECTS, 3 SWS im 5. Semester, 42 Stunden Präsenzunterricht) und das Europarecht (Droit de l'Union Européenne, 4 ECTS, 2 SWS im 5. Semester, 28 Stunden Präsenzunterricht) im 2. oder 3. Studienjahr obligatorisch abgelegt. Damit resultieren 15 ECTS in obligatorischen Fächern.

\section{b. Fremdsprachen}

[42] Auf Bachelorstufe wird ein Kurs Allemand juridique im Umfang von 2 ECTS obligatorisch geprüft. $^{47}$

\section{c. Optionen}

[43] Bereits auf Bachelorstufe können optional verschiedene Spezialkurse im Völkerrecht (z.B. IHR, Völkerstrafrecht etc.) aber auch im vergleichenden Privatrecht (z.B. Droit civil européen: les principes de la responsabilité civile) belegt werden. Insbesondere auf Masterstufe wird zudem ein besonders grosses Angebot an internationalen Fächern angeboten. Daneben bestehen zahlreiche Weiterbildungsangebote auf Nachdiplomstufe.

\section{Université de Neuchâtel (UNINE) - Faculté de droit}

\section{a. Pflichtkurse}

[44] An der Universität Neuenburg ${ }^{48}$ sind im zweiten Studienjahr das Völkerrecht (Droit international public, 2 SWS, insgesamt 56 Stunden Präsenzunterricht, 6 ECTS) und im dritten Studienjahr (6. Semester) das Europarecht (Droit européen institutionnel, 4 SWS, insgesamt 56 Stunden Präsenzunterricht, 6 ECTS) sowie das Internationale Privatrecht (Droit international privé, 2 SWS, insgesamt 56 Stunden Präsenzunterricht, 6 ECTS) Pflicht. Damit werden 18 ECTS obligatorisch internationalen Fächern gewidmet.

\section{b. Fremdsprachen}

[45] Im zweiten Studiensemester muss zudem ein Kurs Terminologie juridique allemande besucht werden (3 ECTS, 2 SWS, 28 Stunden Präsenzunterricht).

\footnotetext{
46 Vgl. Université de Genève, Faculté de droit, Plan d'études (2019-20): (https://www.unige.ch/droit/etudiants /programme-et-calendriers/plan-etudes/) (abgerufen: 29. April 2020).

47 Vgl. Université de Genève, Faculté de droit, Bachelor: http://www.unige.ch/droit/etudes/formation/bachelor.html (abgerufen: 29. April 2020).

48 Vgl. Université de Neuchâtel, Faculté de droit et de sciences économiques, Bachelor: http://www10.unine.ch/ descriptifs/faculte-de-droit/bachelors/bachelor-en-droit/ (abgerufen: 29. April 2020).
} 
c. Optionen

[46] Insbesondere auf Masterstufe wird auch hier ein recht ansprechendes Angebot an internationalen Fächern angeboten.

\section{Universität Basel (UNIBAS) - Juristische Fakultät \\ a. Pflichtkurse}

[47] An der Universität Basel ${ }^{49}$ wird im 5. Semester obligatorisch ein Kurs «Völker- und Europarecht» (6 ECTS, 4 SWS, 56 Stunden Präsenzunterricht) absolviert. Rechtsvergleichung und Internationales Privatrecht werden nicht angeboten bzw. geprüft.

\section{b. Fremdsprachen}

[48] Es besteht kein Pflichtunterricht in juristischer Fremdsprachenterminologie.

\section{c. Optionen}

[49] Im Rahmen der als Wahlpflichtfächer ausgestalteten Grundlagen des «Rechts» werden für BLaw-Studierende auch die zwei Kurse «Die grossen Rechtssysteme aus der Sicht des öffentlichen Rechts» ${ }^{50}$ und «Die grossen Rechtssysteme aus der Sicht des Privatrechts» angeboten. An ihrer Stelle können aber auch andere Kurse besucht werden. Insbesondere auf Masterstufe wird aber ein grosses Angebot an internationalen Fächern angeboten.

\section{Fernuniversität Schweiz und UniDistance (Brig) \\ a. Pflichtkurse}

[50] Seit einigen Jahren bietet auch die Fernuniversität Schweiz bzw. UniDistance ${ }^{51}$ eine Ausbildung zum Juristen an (BLaw in beiden Sprachen, MLaw bisher nur auf Deutsch). Im Bachelor werden keine eigenständigen Kurse in den hier untersuchten internationalen Fächern angeboten. Dies wird damit begründet, dass man diesen Aspekten im Rahmen der übrigen Kurse Raum einräume. ${ }^{52}$ Gewisse Universitäten lassen daher diese Bachelorstudenten nur mit Auflagen zu, wobei nicht sichergestellt ist, dass dabei die fehlenden Kenntnisse internationaler Aspekte des Rechts

49 Vgl.: Ordnung für das Bachelorstudium Rechtswissenschaft der Juristischen Fakultät der Universität Basel vom 1. Dezember 2011: https://www.unibas.ch/dam/Oeffentliche-Dokumente/Rechtserlasse_LegalRegulations/ JuristischeFakultaet_FacultyofLaw/Bachelor_Bachelor/446_210_03/446_210_04.pdf und https://ius.unibas.ch/ fileadmin/user_upload/ius/05_Studium/03_Studieninformationen/01_Bachelorstudium/BA_Bachelorordnung. pdf/ (abgerufen: 29. April 2020).

50 Vgl. Modul: Grundlagen des Rechts (Bachelorstudium: Rechtswissenschaft). Ich danke Prof. Stephan Breitenmoser für diesen Hinweis.

51 Vgl. Fernuniversität Schweiz, Brig - Module: https://unidistance.ch/droit/bachelor/modules/ (abgerufen: 29. April 2020).

52 Persönliche Auskunft des Verantwortlichen Prof. Adriano Previtali (vom 25. Januar 2019). 
kompensiert werden. ${ }^{53}$ Erst auf Masterstufe (die bisher nur auf Deutsch angeboten wird) besteht immerhin ein Pflichtmodul «Völkerrecht» (10 ECTS). ${ }^{54}$

\section{b. $\quad$ Fremdsprachen}

[51] Es besteht kein Obligatorium.

[52] Die Studenten haben auf Bachelorstufe die Möglichkeit, die einzelnen Module nach freier Wahl entweder in deutscher oder französischer Sprache zu absolvieren. Wenn 60\% der Module in der Hauptsprache (Deutsch oder Französisch) und 40\% in der zweiten Sprache belegt werden, erhalten die Studenten einen Bachelor-Abschluss mit dem Zusatz «zweisprachig». Ein Obligatorium besteht aber nicht.

\section{c. Optionen}

[53] Im Master können zudem die Wahlmodule «Internationales Privatrecht» (M 05, 10 ECTS) und/oder «Europarecht» (M13, 10 ECTS) belegt werden.

\section{Zürcher Fachhochschule - ZHAW (Winterthur)}

a. Pflichtkurse

[54] An der Zürcher Fachhochschule (ZHAW = Zürcher Hochschule für Angewandte Wissenschaften $)^{55}$ wird ein «Bachelor (BSc) in Wirtschaftsrecht» angeboten. Es besteht teilweise die Möglichkeit, mit Auflagen in ein MLaw Programm einer Universität aufgenommen zu werden (insbesondere Universität Luzern ${ }^{56}$ ). Andere Universitäten rechnen Leistungen an (u.a. Universität Zürich ${ }^{57}$ oder Neuenburg ${ }^{58}$ ). In Winterthur wird im dritten Semester ein Kurs Public E Private International Law (6 ECTS) geprüft. Hinzu kommen im 4. Semester ebenfalls obligatorische Kurse European Law (6 ECTS) und ein Kurs Anglo-American Law (3 ECTS). Diese werden alle nur auf Englisch angeboten. Es besteht damit ein relevantes Pflichtprogramm von 18 ECTS.

53 An der Universität Zürich werden Studierende mit einem BLaw der Fernuniversität Brig zum Studiengang MLaw UZH normalerweise mit Auflagen im Umfang von 6 ECTS Credits zugelassen (vgl.: https://www.ius.uzh.ch/de/ studies/master/admission.html) (abgerufen: 29. April 2020).

54 Vgl. Fernuniversität Schweiz, Brig, Master-Studium Recht: https://www.ausbildung-weiterbildung.ch/ SchuleFrame.aspx?schoolID=2180 (abgerufen: 29. April 2020).

55 Vgl. ZHAW, Modultafel: https://filestore.sml.zhaw.ch/modultafeln/bachelor/14_modultafel_bl_vz.html (abgerufen: 29. April 2020).

56 Studien- und Prüfungsordnung StuPO 2016 und Wegleitung Zulassung zum Masterstudium unter Auflage [«Passerelle»]: https://www.unilu.ch/studium/lehrveranstaltungen-pruefungen-reglemente $/ \mathrm{rf} / \mathrm{reglemente/ \# section=}$ c34921 (abgerufen: 29. April 2020).

57 Vgl. Universität Zürich: Rechtswissenschaftliche Fakultät, Zulassung: https://www.ius.uzh.ch/de/studies/general/ admission.html.

58 Vgl. Université de Neuchâtel, Faculté de droit et de sciences économiques https://www.unine.ch/droit/en/home/ formations/master.html\#cid1da725dc-71a1-4809-b5e9-b7ec7fbf2797 (abgerufen: 29. April 2020). 


\section{b. Fremdsprachen}

[55] Im Bachelor müssen neben den ohnehin englischen Kursen, die bereits erwähnt wurden, insgesamt 12 ECTS in Legal English absolviert werden (4 Kurse zu 3 ECTS). Hinzu kommen obligatorische Kurse in «Sprache und Recht» (3 ECTS) und «Kommunikation und Recht» (3 ECTS).

\section{c. Optionen}

[56] Im Bachelor müssen gewisse Wahlpflichtmodule (insgesamt 12 ECTS) absolviert werden. Dabei bestehen Optionen in den relevanten Fächern (z.B. Internationales Steuerrecht oder «International Trade and Policy»), die aber auch vermieden werden können. Es besteht u.a. ein Master (MSc) in Management and Law.

\section{HES-SO - HEG Arc (Neuenburg)}

a. Pflichtkurse

[57] Auch für die französische Schweiz bietet die FH der Westschweiz (HES-SO) durch ihre HEG Arc (Neuchâtel Berne Jura) in Neuenburg einen Bachelor of Science in Wirtschaftsrecht an (BSc HES-SO en Droit économique - Business Law - 180 ECTS).$^{59}$ Auch diese Ausbildung kann z.B. in Neuenburg explizit zur Anerkennung gewisser Leistungen bei der Zulassung zum BLaw-Studium führen. ${ }^{60}$ Die Hälfte der Vorlesungen ist juristischen Fächern gewidmet. ${ }^{61}$ Im Modul 5 (des Blocks «Droit» sind zwei Kurse (Droit international 1: Droit européen und Droit international 2 : DIP mit je 2 ECTS) international ausgerichtet (je einer im 1. und 2. Semester). Hinzu kommen ein Kurs Droit international 3 : Droit international privé (2 ECTS) im 4. Semester und ein Kurs Common Law (auf Englisch - 4 ECTS) ebenfalls im 4. Semester. Ausserdem wird im 3. Semester ein Kurs Libre circulation (Binnenmarktrecht der EU bzw. EU/CH - 1 ECTS) als Teil des Moduls Droit public 2 obligatorisch unterrichtet. Damit werden wohl 11 ECTS den internationalen Aspekten eingeräumt.

\section{b. $\quad$ Fremdsprachen}

[58] Auf die obligatorische Fremdsprachenausbildung wird relativ grosses Gewicht gelegt. Im Block Communication werden im Modul 2 zwei Kurse Deutsch (Allemand 1 mit 2 ECTS und Allemand 2 mit 3 ECTS) und zwei Kurse Englisch (Anglais 1 mit 3 ECTS und Anglais 2 mit 2 ECTS) verlangt (1. und 2. Semester). Hinzu kommen noch die Kurse Allemand juridique (2 ECTS) und Anglais juridique (2 ECTS) im 3. Semester sowie ein Kurs Anglais et allemand commercial 2 ECTS) im 4. Semester. Dies bedeutet, dass mindestens 16 ECTS der Fremdsprache Englisch bzw. der zweiten Landessprache Deutsch gewidmet sind.

59 Der Verantwortliche ist Pierre-Yves Lavanchy. Vgl. https://www.hes-so.ch/fr/bachelor-droit-economique-1463. html? theme=T8.

60 Vgl. Université de Neuchâtel, Faculté de droit et de sciences économiques, Master: https://www.unine.ch/droit/en/ home/formations/master.html\#cid1da725dc-71a1-4809-b5e9-b7ec7fbf2797 (abgerufen: 29. April 2020).

61 Vgl. HE-Arc, Plan d'études (https://www.he-arc.ch/gestion/bachelor-bl) und Modulbeschreibungen (https://www. he-arc.ch/reglementation) (abgerufen: 29. April 2020). 
c. Optionen

[59] Gewisse der Optionen (Insbesondere im 5. und 6. Semester) enthalten auch internationale Elemente. Zurzeit wird noch kein konsekutiver Master angeboten. Hingegen besteht ein Angebot im Rahmen der Weiterbildung zum MAS Lutte contre la criminalité économique.

\section{Kalaidos Fachhochschule Schweiz (Zürich)}

\section{a. Pflichtkurse}

[60] An der privaten Kalaidos Fachhochschule Schweiz wird seit geraumer Zeit ebenfalls ein Bachelor of Science FH in Wirtschaftsrecht (Business Law) angeboten. Hierbei wird im Grundstudium (1. und 2. Jahr) in einem Modul Privatrecht 2 (6 ECTS) auch das Wiener Kaufrecht behandelt. In der Einführung in die Rechtswissenschaft (6 ECTS) wird neben vielen anderen Themen der Bereich «Nationales Recht und internationale Rechtsordnungen: Völkerrecht, Europarecht, überstaatliche Garantie der Menschenrechte, Gerichtsbarkeit» vermittelt. Auch in der Vorlesung Compliance (6 ECTS) im Hauptstudium (3. und 4. Jahr) wird das Thema «Nationale und internationale rechtliche Rahmenbedingungen» erwähnt Aufgrund der für die anderen Institutionen verwendeten Systematik (insbesondere Fernstudien Schweiz/Unidistance, aber allgemein auch für die Vorlesungen mit schwerpunktmässig innerstaatlichen Inhalten) erscheint hier die Schwelle zur Feststellung von eigentlichem garantiertem Unterricht in internationalen Aspekten nicht erreicht. Im Rahmen der «Fallbearbeitung im öffentlichen Recht III» (6 ECTS) werden hingegen auch Fälle im Völkerrecht ${ }^{62}$ behandelt (2 ECTS). ${ }^{63}$ Damit resultieren insgesamt für diese Ausbildung 2 ECTS.

[61] Hier werden neuerdings auch ein Bachelor in Recht (Bachelor of Arts in Law Kalaidos FH, 180 ECTS) und ein Master in Recht (Master of Arts in Law Kalaidos FH, 90 ECTS ${ }^{64}$ angeboten. Es besteht keine Präsenzpflicht. Dabei wird im dritten Studienjahr des Bachelors ein Kurs «Völkerrecht» (6 ECTS) geprüft, der sowohl die Grundlagen des Europarechts als auch des Völkerrechts behandelt. Im Rahmen der «Fallbearbeitung im öffentlichen Recht III» (6 ECTS) werden auch hier Fälle im Völkerrecht behandelt (2 ECTS), womit ungefähr 8 ECTS internationalen Aspekten gewidmet werden.

\section{b. Fremdsprachen}

[62] Es besteht kein Obligatorium.

\footnotetext{
62 Vgl. Kalaidos FH, Recht: https://www.kalaidos-fh.ch/de-CH/Lawschool/Studium/Bachelor-of-Law/ Hauptstudium (abgerufen: 29. April 2020) und persönliche Auskunft der Modulverantwortlichen Prof. Patricia Egli (30. März 2020).

63 See Kalaidos FH, Recht: https://www.kalaidos-fh.ch/de-CH/Prorektorat-Lehre/BBA-WR\#fakten (abgerufen: 29. April 2020).

64 Der Titel wird von der Institution auch als «Master in Law» (MLaw) beworben; vgl. https://www.kalaidos-fh.ch/ de-CH/Lawschool/Studium/Master-of-Law/ (abgerufen: 29. April 2020).
} 
c.

Optionen

[63] Auf Masterstufe (Master of Arts in Law Kalaidos FH) werden weitere Module zu internationalen Aspekten angeboten, zurzeit Internationales Privat- und Verfahrensrecht (6 ECTS) und International Contract Law (3 ECTS) ${ }^{65}$ Daneben werden spezifische Weiterbildungsangebote (MAS) im (internationalen) Steuerrecht angeboten. ${ }^{66}$ Für Absolventen des eigenen MSc in Wirtschaftsrecht ${ }^{67}$ oder desjenigen der ZHAW ${ }^{68}$ wird eine Passerelle angeboten. Für die Absolventen des eigenen BSc ist das insofern relevant bezüglich der hier behandelten Fragestellung, als dabei die erwähnten Kurse Internationales Privat- und Verfahrensrecht (6 ECTS) ebenfalls Pflicht darstellen und der Kurs Völkerrecht des Bachelors (6 ECTS) des eigenen BLaw-Programms nachgeholt wird. Da dies aber erst auf Masterebene vorgesehen ist, fliesst es ebenfalls nicht als garantiert in unsere Erhebung als solche ein.

\section{Analyse und Postulate}

[64] Insgesamt kann man die Ergebnisse der vorliegenden Übersicht für die obligatorischen international ausgerichteten Leistungen in der traditionellen Juristenausbildung (BLaw und MLaw) in der Schweiz wie folgt zusammenfassen:

1. Es bestehen erhebliche Unterschiede bezüglich der Ausbildung in internationalen Aspekten zwischen Bachelor-Absolventen (180 ECTS) in der Schweiz.

Während etwa die Universität Lausanne bereits auf der Grundstufe obligatorisch 20 ECTS in den relevanten Fächern und 3 ECTS für die deutsche Rechtssprache vorsieht, kann man einen BLaw der UniDistance erwerben, ohne irgendeine Leistung diesbezüglich erbringen zu müssen (0 ECTS). An den meisten Deutschschweizer Universitäten (Basel, Bern, Luzern, St. Gallen ${ }^{69}$ ) wird zumeist nur ein Pensum von 6-9 ECTS in Völker- und Europarecht angeboten. Zürich legt mit der Studienreform auf 15 ECTS zu und schliesst damit zu Genf auf. Neuchâtel pflegt die französischsprachige Tradition mit 18 ECTS, während auch der französischsprachige Lehrgang in Freiburg nur 9 ECTS vorsieht (wie der deutschsprachige). Die Fernuniversität Schweiz bietet zwar 10 ECTS an, da diese aber erst auf Masterstufe erlangt werden, besteht hier die Gefahr der totalen Vermeidung bei Fakultätswechsel. Somit gewähren beide Fernuniversitätslehrgänge keinerlei Garantie, dass internationale Aspekte im BLaw geprüft wurden. Es besteht sicherlich kein gemeinsames schweizerisches Konzept. Gewisse Institutionen sollten dringend ihre Minimalanforderungen überdenken. Die Bachelorangebote der Fachhochschulen (BA und BSc) liegen hingegen im Durchschnitt (BA,

65 Vgl. Kalaidos FH, Recht: https://www.kalaidos-fh.ch/de-CH/Lawschool/Studium/Master-of-Law/Module (abgerufen: 29. April 2020).

66 Vgl. Kalaidos FH, Recht: https://www.kalaidos-fh.ch/de-CH/Lawschool/Weiterbildung/Weiterbildung-MAS-LLM (abgerufen: 29. April 2020).

67 Vgl. oben, Kosten: Für InhaberInnnen eines BSc in Wirtschaftsrecht der Kalaidos FH (das Studium umfasst 111 ECTS-Punkte): CHF 8'166 pro Semester oder CHF 1'361 pro Monat. Inkl. Prüfungsgebühren (erster Versuch), Übungsmaterial und Begleithefte. Zzgl. einer ein-maligen Immatrikulationsgebühr von CHF 100.

68 Kosten: Für InhaberInnnen eines BSc in Wirtschaftsrecht der ZHAW (das Studium umfasst 144 ECTS-Punkte) CHF 9'600 pro Semester oder CHF 1'600 pro Monat. Inkl. Prüfungsgebühren (erster Versuch), Übungsmaterial und Begleithefte. Zzgl. einer einmaligen Immatrikulationsgebühr von CHF 100. 
Kalaidos FH) oder gehören sogar zur Spitzengruppe (BSc, ZHAW und HES-SO) bezüglich Internationalität.

Mit 9-12 ECTS und eher mehr Präsenzunterricht in diesen Fächern lassen die Westschweizer Universitäten (inkl. Freiburg) die Deutschschweizer Universitäten (Basel, Bern, Luzern) klar hinter sich. Der Zeitpunkt für den Unterricht spielt bei der Beurteilung der Anzahl ECTS und Präsenzunterrichtsstunden wohl eine eher untergeordnete Rolle. St. Gallen kann durch den Sprachunterricht ins Mittelfeld aufschliessen. Allerdings ist der Umfang des Unterrichtes im BLE-Programm (auf Bachelorstufe) klar im unteren Bereich.

Die Schweizerische Vereinigung für internationales Recht (SVIR) sollte sich dringend mit den Mindestanforderungen in diesem Bereich beschäftigen und entsprechende Empfehlungen an die relevanten Einrichtungen richten.

2. Das Völker- und Europarecht wird fast immer auf Bachelorstufe angeboten, aber es ist nicht garantiert. Beim Wechsel der Institution kann es aufgrund von mangelnder Einheitlichkeit zu fehlenden Kenntnissen der internationalen Aspekte kommen.

Das Völker- und Europarecht wird fast immer auf Bachelorstufe angeboten. Nur die Fernuniversität Schweiz/UniDistance vermittelt keine ausgewiesenen Kenntnisse auf Bachelorstufe in diesem Bereich. Auch die Universität St. Gallen verzichtet bei ihrem BLE (BA HSG in Rechtswissenschaft mit Wirtschaftswissenschaften), der wie ein BLaw den Zugang zum Anwaltspraktikum garantieren soll, ganz auf Vorlesungen im «Völker- und Europarecht». ${ }^{70}$ Dies kann bei diesen Institutionen sehr leicht dazu führen, dass die Studierenden im Laufe ihrer Ausbildung überhaupt keine Kenntnisse auf diesem Gebiet erwerben (denn auch auf Masterstufe beseht diesbezüglich wie gezeigt keine Garantie, wenn Studenten nicht das darauf abgestimmte Folgeprogramm belegen).

Es wäre daher wünschenswert, dass auch an der Universität St. Gallen (BLE-Programm) und an der Fernuniversität Schweiz/UniDistance (Brig) obligatorisch Kenntnisse im Völker- und Europarecht auf Bachelorstufe vermittelt werden. Bis dahin ist den anderen Universitäten bei der Zulassung zum MLaw-Studium zu empfehlen, fehlende Kenntnisse in der Form von Auflagen einzufordern.

3. Internationales Privatrecht ist selten Pflichtfach auf Bachelorstufe

Das IPR wird in weniger als der Hälfte der Universitäten als Pflichtstoff im BLaw geprüft (3-6 ECTS). Einerseits sind es die rein französischsprachigen Universitäten (Genf, Lausanne, und Neuenburg) andererseits jene, die eine wirtschaftliche Ausrichtung anstreben (St. Gallen, Zürich). Letzteres gilt auch für die Ausbildung an der ZHAW (FH Winterthur). Es wäre wünschenswert, dass in der privatrechtlichen Ausbildung im Bachelor internationale Aspekte eingebaut werden. Dies sollte Aspekte des IPR und eventuell auch der Rechtsvergleichung berücksichtigen. Wo dies nicht in einem eigenen Kurs stattfindet, sollte sichergestellt werden, dass die Beachtung dieser Aspekte transparent und nachprüfbar in den bestehenden Einheiten ausgewiesen wird.

70 Die angedachte Studienreform 2021 könnte hier zu einer Verbesserung führen, wobei sichergestellt werden sollte, dass die Grundkenntniss in Völker- und Europarecht auf jeen Fall von allen Studenten erworben werden. 
4. Völker- und Europarecht werden vom Umfang her praktisch gleich unterrichtet

Es zeigt sich, dass trotz der späteren Entwicklung des Europarechts zum eigenen Studienfach der Unterricht heute mit dem Völkerrecht praktisch gleichziehen konnte. Oft wird der Unterricht ohnehin in einer gemeinsamen Veranstaltung (aber allenfalls mit unterschiedlichen Dozenten) kombiniert. Nur Genf gibt dem internationalen Recht leicht mehr Raum als dem Europarecht (4 gegenüber 6 ECTS).

5. Rechtsvergleichung und rechtsvergleichende Vorlesungen können einfach vermieden werden

Ausser in Lausanne wird der Rechtsvergleichung nirgends (mehr) ein obligatorischer Charakter eingeräumt. Selbst hier beschränkt sich dieser auf die Privatrechtsvergleichung, was sicher der Hochschätzung der langen Tradition und der Präsenz des Schweizerischen Instituts für Rechtsvergleichung (SIRV, Lausanne) zu verdanken ist.

6. Deutsch wird an den meisten französischsprachigen Institutionen obligatorisch (in geringem Umfang) angeboten

An den rein französischsprachigen Universitäten der Schweiz (mit Ausnahme von UniDistance) wird (in geringem Umfang) deutsche Rechtsterminologie unterrichtet (2-3 ECTS). In Fribourg setzt man auf die indirekte Wirkung der Zweisprachigkeit.

UniDistance sollte einen obligatorischen Unterricht in deutscher Rechtssprache erwägen.

7. Französisch ist an keiner der deutschsprachigen Institutionen obligatorisch

An den deutschsprachigen Universitäten (inklusive deutschsprachige Ausbildung in Freiburg) sieht man es nicht als notwendig an, obligatorisch ein Verständnis der französischen Rechtssprache zu fordern. Darin spiegeln sich klar die Grössenverhältnisse der Sprachgruppen in der Schweiz. In St. Gallen besteht zwar ein Obligatorium für 4 ECTS in Fremdsprachen. Es muss sich aber weder um Französisch handeln, noch ist Rechtsterminologie vorgesehen. Immerhin empfiehlt die Universität St. Gallen aber entweder den Kurs Le français $d u$ droit oder alternativ English for Law im Rahmen dieser Pflichtveranstaltung zu absolvieren. Es wäre wünschenswert, dass auch in der Deutschschweiz das Verständnis französischsprachiger Literatur und des Fallrechts gewährleistet wird.

8. Englisch oder spezifische englische Rechtsterminologie fehlt in der obligatorischen Ausbildung

Bisher wird zur Erlangung eines BLaw in der Schweiz an keiner universitären Institution verlangt, dass sich die Studierenden über Englischkenntnisse ausweisen. In St. Gallen besteht zwar ein Obligatorium für 4 ECTS in Fremdsprachen. Es muss sich aber weder um Englisch handeln, noch ist Rechtsterminologie verpflichtend. Immerhin empfiehlt die Universität St. Gallen aber entweder den Kurs Le français du droit oder alternativ English for Law im Rahmen dieser Pflichtveranstaltung zu absolvieren. Die ZHAW und die HES-SO gehen hier bedeutend weiter, in dem sie spezifische Leistungen in «Legal English» und englischsprachige Pflichtvorlesungen prüfen.

Alle Institutionen sollten erwägen, Kenntnisse in Legal English zu gewährleisten.

9. Im optionalen Bereich glänzen v.a. die grösseren Institutionen in der Schweiz mit einem umfassenden Angebot an fremdsprachigen und internationalen Kursen 
Traditionell ist das fakultative Angebot an Kursen im Völkerrecht, Europarecht und dem internationalen Privatrecht gut. Selbst in der Rechtsvergleichung können die meisten traditionellen Fakultäten etwas anbieten. Zudem sind in den letzten Jahren zunehmend Weiterbildungsangebote (zumeist auf Englisch) entstanden. An zahlreichen Institutionen bestehen diese im Wirtschaftsrecht, zudem in Genf (und andernorts in geringerem Umfang) im Bereich des humanitären Völkerrechts und der Menschenrechte. Die Programme im Europarecht haben eher an Bedeutung abgenommen.

Es wäre empfehlenswert, die Ressourcen, die für fakultative Angebote gebunden sind, auch im obligatorischen Bereich besser zu nutzen und sicherzustellen, dass die internationalen Aspekte als fundamentaler Bestandteil der Grundausbildung (Bachelor) aller Juristen garantiert werden.

10. Neuere Anbieter (Fachhochschulen, Wirtschafts- und sozialwissenschaftliche Einheiten) sind wesentlich innovativer bei der Einführung von internationalrechtlich relevanten Angeboten

Wie auch in anderen Bereichen ist die juristische Ausbildung in der Schweiz neuen Strömungen ausgesetzt. Während die klassische Ausbildung (BLaw und MLaw) noch vorherrschen, bieten zahlreiche Universitäten auch Bachelor- und v.a. Masterprogamme mit einem stärker interdisziplinären Charakter (insbesondere Recht mit Wirtschaftswissenschaften) an. Auch Masterprogramme mit stark internationalem Charakter (z.B. MIL St. Gallen oder MIL IHEID) sind hier im Zunehmen begriffen. Während diese Programme das Spektrum an Ausbildungsmöglichkeiten und damit Ausbildungsprofilen erweitern, nimmt gleichzeitig natürlich die Übersichtlichkeit ab.

Hinzu kommen v.a. noch die Angebote von Fachhochschulen, die für die Schweiz ebenfalls neu sind. Hier stellt sich grundsätzlich die Frage der genügenden Ausbildung in internationalen Aspekten des Rechts ebenfalls. Es erscheint, dass hierbei einige Programme ganz klar auf Internationalisierung setzen. Diesen Programmen fehlt aber dann normalerweise der notwendige Anteil an innerstaatlichem Recht, um ohne weiteres traditionelle juristische Funktionen wahrzunehmen.

Umso wichtiger wird eine genaue Analyse des Profils eines Bewerbers im Hinblick auf die angestrebte Tätigkeit. Im Hinblick auf die Zulassung zu regulierten Berufen (Anwalt, Notar, Staatsanwalt, Richter etc.) ist diese Problematik bereits bekannt, wird aber auch hier in den nächsten Jahren zusätzliche Klärung benötigen. Die Fachvereinigungen (Schweizerische Vereinigung für internationales Recht, Schweizerische Vereinigung für Europarecht, Schweizerischer Juristenverein etc.) sind eingeladen, sich hier verstärkt zu engagieren.

11. Die Konkretisierung der internationalen Aspekte in Bezug auf das schweizerische Recht wird nicht immer sichergestellt

Eine besondere Problematik besteht darin, dass die internationalen Aspekte des Rechts bezüglich ihrer Bedeutung für das schweizerische Recht konkretisiert werden müssen. Das wird nicht immer sichergestellt. Dies kann einerseits passieren, wenn beim Unterricht in der Schweiz der Bezug zum positiven Recht der Schweiz (und insbesondere der Rechtsprechung) nicht hergestellt wird. Ausserdem ist der Erwerb internationaler Aspekte des Rechts im Ausland zwar grundsätzlich sehr wünschenswert und positiv zu bewerten, garantiert aber ebenfalls nicht das Verständnis, wie die schweizerische Rechtsordnung genau mit dem Völkerrecht oder Europarecht umgeht (Rang, unmittelbare Anwendbarkeit, Berücksichtigung der Rechtsprechung des EuGH etc.), oder wie das schweizerische IPR oder 
IZPR sich darstellt (z.B. ordre public in der Rechtsprechung des Bundesgerichts oder Fragen des genügenden Binnenbezugs bei der Anerkennung von ausländischen Entscheiden oder Schiedsurteilen).

Es muss sichergestellt werden, dass die internationalen Aspekte in Bezug auf das schweizerische Recht konkretisiert werden. Die Ausbildungsinstitutionen müssen sicherstellen, dass beim Unterricht und bei der Anerkennung von im Ausland erbrachten Leistungen dieser Problematik Rechnung getragen wird.

12. Garantie der Qualität und Transparenz an allen Institutionen, die an der juristischen Ausbildung beteiligt sind - Formulierung eines Konzepts

Traditionell wird auch in der Schweiz (zu Recht) grosses Gewicht auf die akademische Freiheit gelegt. Bezüglich der traditionellen Universitäten wurde die Qualität des Unterrichts normalerweise über die Reputation und die informelle gegenseitige Kontrolle (Peer review) sichergestellt. Normalerweise sind es die innerstaatlichen und internationalen Fachvereinigungen, die hier besonders gefordert sind. Die Leitungen der betroffenen Institutionen sollten interessiert sein, ihre eigene Qualität so sicherzustellen. Aufgrund der vielen neuen Akteure und der neuen Organisationsformen, ist dies heute nicht immer möglich.

Die schweizerischen Fachvereinigungen (insbesondere SVIR und SVER) wären gut beraten, sicherzustellen, dass sie für alle Dozenten in den betroffenen Fächern interessant und relevant sind. Die Universitäten sollten eingeladen werden, sicherzustellen, dass die betroffenen Fachgebiete genügend ausgestattet werden und dabei klare Verantwortlichkeiten und Transparenz geschaffen werden. Es wäre wünschenswert, dass die akkreditierten Institutionen ein Konzept ${ }^{71}$ nicht nur ihrer Ausbildung insgesamt oder für einzelne Lehrgänge erstellen, sondern auch bezüglich der angemessenen Berücksichtigung internationaler Aspekte.

Prof. Dr. Andreas R. Ziegler, Professor für internationales Recht am Centre de droit comparé, européen et international (CDCEI) und Direktor des LLM International Business Law an der Ecole de droit der Universität Lausanne (UNIL). Er ist Präsident der Schweizer Sektion der International Law Association (ILA) und Vorstandsmitglied der Schweizerischen Vereinigung für internationales Recht (SVIR) und der Deutschen Gesellschaft für internationales Recht (DGIR).

71 Zu den (zumeist sehr rudimentären) Ausbildungskonzepten von einigen, wenigen der behandelten universitären Einrichtungen vgl. Sethe (Fn. 2), S. 25-28. 